\title{
A Symmetrization Result for Nonlinear Elliptic Equations
}

\author{
Vincenzo Ferone and Basilio Messano
}

\author{
Dipartimento di Matematica e Applicazioni \\ "R. Caccioppoli" \\ Università di Napoli "Federico II" \\ Complesso Univ. Monte S. Angelo \\ Via Cintia - 80126 Napoli - Italy \\ ferone@unina.it
}

\author{
Dipartimento di Matematica e Applicazioni \\ "R.Caccioppoli" \\ Università di Napoli "Federico II" \\ Via Claudio, 21 - 80125 Napoli - Italy \\ messano@unina.it
}

Recibido: 21 de Febrero de 2003

Aceptado: 30 de Septiembre de 2003

\begin{abstract}
We consider a solution $u$ of the homogeneous Dirichlet problem for a class of nonlinear elliptic equations in the form $A(u)=g(x, u)+f$, where the principal term is a Leray-Lions operator defined on $W_{0}^{1, p}(\Omega)$. The function $g(x, u)$ satisfies suitable growth assumptions, but no sign hypothesis on it is assumed. We prove that the rearrangement of $u$ can be estimated by the solution of a problem whose data are radially symmetric.
\end{abstract}

Key words: symmetrization, rearrangement, nonlinear elliptic PDE's

2000 Mathematics Subject Classification: 35J65, 35B45

\section{Introduction}

Let $\Omega$ be a bounded open set of $\mathbf{R}^{n}$. We consider solutions to Dirichlet problems whose prototype can be written as follows:

$$
\begin{cases}-\Delta_{p} u=c|u|^{p-2} u+f & \text { in } \Omega \\ u=0 & \text { on } \partial \Omega\end{cases}
$$

where $\Delta_{p}$ is the $p$-laplacian operator $\left(\Delta_{p} u=\operatorname{div}\left(|D u|^{p-2} D u\right)\right), p>1$, and $c, f$ are bounded functions. It is well known that symmetrization techniques allow to estimate 
a solution $u$ to problem (1) by means of the solution to a problem whose data are spherically symmetric. In the case $c(x) \leq 0$ the comparison has been made in two different ways. To be more precise, if $w$ solves the problem:

$$
\begin{cases}-\Delta_{p} w=f^{\#} & \text { in } \Omega^{\#} \\ w=0 & \text { on } \partial \Omega^{\#}\end{cases}
$$

where $\Omega^{\#}$ is the ball centered at the origin, having the same measure as $\Omega$, and $f^{\#}$ is the spherically symmetric decreasing rearrangement of $f$, then (see [19]):

$$
u^{*}(s) \leq w^{*}(s), \quad s \in[0,|\Omega|]
$$

where $u^{*}$ and $w^{*}$ are the decreasing rearrangements of $u$ and $w$, respectively (see, Section 2 for precise definitions).

On the other hand, if $z$ solves the problem:

$$
\begin{cases}-\Delta_{p} z=-c_{\#}|z|^{p-2} z+f^{\#} & \text { in } \Omega^{\#} \\ z=0 & \text { on } \partial \Omega^{\#}\end{cases}
$$

where $c_{\#}$ is the spherically increasing rearrangement of $c$, then (see [11]):

$$
\int_{0}^{s}\left(u^{*}(t)\right)^{p-1} d t \leq \int_{0}^{s}\left(z^{*}(t)\right)^{p-1} d t, \quad \forall s \in[0,|\Omega|] .
$$

In the present paper, we are interested in studying what happens when no sign assumption is made on $c$. In this case we can compare a solution of (1) with the solution of the problem:

$$
\begin{cases}-\Delta_{p} v=\hat{c}|v|^{p-2} v+f^{\#} & \text { in } \Omega^{\#}, \\ v=0 & \text { on } \partial \Omega^{\#}\end{cases}
$$

where $\hat{c}$ has to be built using the rearrangements of the positive and negative parts of $c$, i. e., $c^{+}(x)=\max \{c(x), 0\}, c^{-}(x)=\max \{-c(x), 0\}$, that is $\hat{c}(x)=\left(c^{+}\right)^{\#}(x)-$ $\left(c^{-}\right)_{\#}(x)$.

The fact that no sign assumption is made on $c$ forces us to require that $c^{+}$is sufficiently small. More precisely, denoted by $\lambda\left(\Omega^{\#}\right)$ the first eigenvalue of the following Dirichlet problem:

$$
\begin{cases}-\Delta_{p} \psi=\lambda|\psi|^{p-2} \psi & \text { in } \Omega^{\#} \\ \psi=0 & \text { on } \partial \Omega^{\#}\end{cases}
$$

if the condition below is satisfied:

$$
\left\|c^{+}\right\|_{\infty}<\lambda\left(\Omega^{\#}\right)
$$


then the decreasing rearrangement of any solution of (1) can be estimated in terms of the decreasing rearrangement of $v$. So, set $s_{0}=\inf \left\{s \in[0,|\Omega|]:\left(c^{-}\right)_{*}(s)>0\right\}$, the above estimate is pointwise in $\left[0, s_{0}\right]$, i. e., $u^{*}(s) \leq v^{*}(s), \forall s \in\left[0, s_{0}\right]$, while it is given in terms of integrals as in (4) in the interval $\left[s_{0},|\Omega|\right]$ (see Theorem 4.1).

Several results of this type can be found in the literature (see, for example, [3], [7], [8], [9], [11], [16], [18], [19], [20], [22]). In particular, the influence of the lowerorder term has been considered in [3], [8], [9], [11] and, in [3], when $p=2$, no sign assumption is made.

We remark that, being $\left\|c^{+}\right\|_{\infty}=\left\|\hat{c}^{+}\right\|_{\infty}$, assumption (7) implies that problem (5) admits a unique nonnegative radially decreasing symmetric solution $v$ which minimizes a suitable functional (see Theorem 3.1). The above mentioned properties of $v$ are essential in proving the comparison result. On the other hand, if (7) is not verified, then, in general, a comparison result cannot be expected. To this aim, let us consider the linear problem

$$
\begin{cases}-\Delta u=c u+f & \text { in } \Omega \\ u=0 & \text { on } \partial \Omega\end{cases}
$$

with $c>0$ and $f>0$. If $\Omega$ is not a ball, we recall that (see [5], [14], [1]) $\lambda\left(\Omega^{\#}\right)<\lambda(\Omega)$ and then, if $c=\lambda\left(\Omega^{\#}\right)$, problem (8) admits a unique solution, while the symmetrized problem (5) (with $p=2$ ) does not.

The paper is organized as follows. In Section 2 we recall the definitions of rearrangement and some properties used in the next sections. Section 3 is devoted to the study of the properties of the solutions of problems defined in a ball in the form (5). Among the other things, we show that (7) guarantees the existence and uniqueness of the solution of (5), which is a radially symmetric decreasing function. Such a result, together with an integro-differential inequality for the rearrangement of the solution of (1) allows us, in Section 4, to prove the comparison result quoted above. As a matter of fact, such a comparison result is stated for solutions of Dirichlet problems in the following general form:

$$
\begin{cases}-\operatorname{div}(a(x, u, D u))=g(x, u)+f & \text { in } \Omega, \\ u=0 & \text { on } \partial \Omega,\end{cases}
$$

where $a(x, s, \xi)$ and $g(x, s)$ satisfy suitable conditions (see Section 4).

\section{Preliminaries and background information}

In this section we recall the definition of decreasing rearrangement of a measurable function; moreover, we state some integral inequalities which will be useful in the next sections.

Let $\Omega$ be an open bounded subset of $\mathbf{R}^{n}$ and $u: \Omega \rightarrow \mathbf{R}$ be a measurable function. If one denotes by $|E|$ the Lebesgue measure of a set $E \subset \mathbf{R}^{n}$, one can define the 
distribution function $\mu_{u}$ of $u$ as follows:

$$
\mu_{u}(t)=|\{x \in \Omega:|u(x)|>t\}|, \quad t \geq 0 .
$$

The function $\mu_{u}$ is decreasing and right continuous; moreover, its generalized inverse function is the decreasing rearrangement $u^{*}$ of $u$ :

$$
u^{*}(s)=\sup \left\{t \geq 0: \mu_{u}(t)>s\right\}, \quad s \in[0,|\Omega|] .
$$

The spherically symmetric decreasing rearrangement of $u$ is defined by:

$$
u^{\#}(x)=u^{*}\left(\omega_{n}|x|^{n}\right), \quad x \in \Omega^{\#},
$$

where $\Omega^{\#}$ is the ball centered at the origin having the same measure as $\Omega$ and $\omega_{n}$ is the measure of the unit ball in $\mathbf{R}^{n}$.

In addition to the above rearrangements it is useful to consider the increasing rearrangement of $u$, that is the function:

$$
u_{*}(s)=u^{*}(|\Omega|-s), \quad s \in[0,|\Omega|]
$$

likewise we define by:

$$
u_{\#}(x)=u_{*}\left(\omega_{n}|x|^{n}\right), \quad x \in \Omega^{\#},
$$

the spherically symmetric increasing rearrangement of $u$.

For an exhaustive treatment of the properties of rearrangements we refer to [2], $[5],[13],[14],[17]$. We only recall some well known results which will be useful in the next sections.

Lemma 2.1. Let $f_{1}(s)$ and $f_{2}(s)$ be measurable, positive functions such that

$$
\int_{0}^{r} f_{1}(s) d s \leq \int_{0}^{r} f_{2}(s) d s, \quad \forall r \in[0, \delta] .
$$

If $g \geq 0$ is a decreasing function then:

$$
\int_{0}^{r} f_{1}(s) g(s) d s \leq \int_{0}^{r} f_{2}(s) g(s) d s, \quad \forall r \in[0, \delta] .
$$

Lemma 2.2. If $f$ and $g$ belong to $L^{p}(\Omega), 1 \leq p \leq+\infty$, and

$$
\int_{0}^{r} f^{*}(\tau) d \tau \leq \int_{0}^{r} g^{*}(\tau) d \tau, \quad \forall r \in[0,|\Omega|]
$$

then:

$$
\|f\|_{p} \leq\|g\|_{p}
$$


Finally, let us conclude this section by recalling a technical lemma which will be useful in the following (see [15]).

Lemma 2.3. If $p \geq 2$, then:

$$
\left|\xi_{2}\right|^{p} \geq\left|\xi_{1}\right|^{p}+p\left|\xi_{1}\right|^{p-2} \xi_{1}\left(\xi_{2}-\xi_{1}\right)+\frac{\left|\xi_{2}-\xi_{1}\right|^{p}}{2^{p-1}-1}
$$

for every $\xi_{1}, \xi_{2} \in \mathbf{R}^{n}$.

If $1<p<2$, then:

$$
\left|\xi_{2}\right|^{p} \geq\left|\xi_{1}\right|^{p}+p\left|\xi_{1}\right|^{p-2} \xi_{1}\left(\xi_{2}-\xi_{1}\right)+h(p) \frac{\left|\xi_{2}-\xi_{1}\right|^{2}}{\left(\left|\xi_{1}\right|+\left|\xi_{2}\right|\right)^{2-p}},
$$

for every $\xi_{1}, \xi_{2} \in \mathbf{R}^{n}$, where $h(p)$ is a positive constant depending on $p$.

\section{The radial case}

In this section we consider the following Dirichlet problem with radially symmetric data:

$$
\begin{cases}-\Delta_{p} v=b|v|^{p-2} v+f & \text { in } B \\ v=0 & \text { on } \partial B\end{cases}
$$

where $B$ is a ball centered at the origin, $b(x)=b(|x|)$ and $f(x)=f(|x|)$ are bounded radially decreasing functions, with $f(x) \geq 0$.

Theorem 3.1. If $\left\|b^{+}\right\|_{\infty}<\lambda(B)$, where $\lambda(B)$ is the first eigenvalue of problem (6) in $B$, then the problem (9) admits a unique nonnegative solution $v(x)$ such that

$$
v(x)=v^{\#}(x) .
$$

Furthermore, $v$ minimizes the functional

$$
E(w)=\frac{1}{p} \int_{B}|D w|^{p} d x-\frac{1}{p} \int_{B} b|w|^{p} d x-\int_{B} f w d x, \quad w \in W_{0}^{1, p}(B),
$$

that is:

$$
\begin{array}{rl}
\frac{1}{p} \int_{B}|D v|^{p} & d x-\frac{1}{p} \int_{B} b|v|^{p} d x-\int_{B} f v d x \leq \\
\leq & \frac{1}{p} \int_{B}|D w|^{p} d x-\frac{1}{p} \int_{B} b|w|^{p} d x-\int_{B} f w d x, \quad \forall w \in W_{0}^{1, p}(B) .
\end{array}
$$

Proof. We immediately observe that a simple argument based on the variational characterization of $\lambda(B)$,

$$
\lambda(B)=\inf _{\substack{w \in W_{0}^{1, p}(B) \\ w \neq 0}} \frac{\int_{B}|D w|^{p} d x}{\int_{B}|w|^{p} d x},
$$


allows us to say that the theorem is trivial when $f \equiv 0$ in $B$. So, from now on, we will suppose that $f \not \equiv 0$.

The existence of a nonnegative solution $v(x)$ of (9) follows from Theorem 2 of [10] and such a solution minimizes the functional $E$. Moreover, it is easy to see that any solution of (9) is positive in $B$. Indeed, if $v$ solves (9), we can use as a test function its negative part $v^{-}$obtaining:

$$
\int_{B}\left|D v^{-}\right|^{p} d x=\int_{B} b\left|v^{-}\right|^{p} d x-\int_{B} f v^{-} d x<\lambda(B) \int_{B}\left|v^{-}\right|^{p} d x-\int_{B} f v^{-} d x
$$

Assuming $v^{-} \not \equiv 0$ and recalling the characterization of $\lambda(B)$, we immediately obtain a contradiction. Then $v \geq 0$ and, using the maximum principle in [21], one actually gets $v>0$ in $B$.

In order to prove the uniqueness we will follow an argument which can be found in [15] (see also [1], [6], [10]). Let $u$ and $v$ be two positive solutions of (9) such that $u \not \equiv v$. Let us consider the following two test-functions:

$$
\varphi_{1}(x)=\frac{u^{p}-v^{p}}{u^{p-1}}, \quad \varphi_{2}(x)=\frac{v^{p}-u^{p}}{v^{p-1}} .
$$

Well known regularity results allow us to use $\varphi_{1}$ in the equation satisfied by $u$ and $\varphi_{2}$ in the one satisfied by $v$, obtaining:

$$
\begin{array}{r}
\int_{B}\left[|D u|^{p-2} D u\left(D u-p\left(\frac{v}{u}\right)^{p-1} D v+(p-1)\left(\frac{v}{u}\right)^{p} D u\right)\right] d x= \\
=\int_{B}\left(u^{p}-v^{p}\right)\left[b+\frac{f}{u^{p-1}}\right] d x
\end{array}
$$

and

$$
\begin{gathered}
\int_{B}\left[| D v | ^ { p - 2 } D v \left(D v-p\left(\frac{u}{v}\right)^{p-1}\right.\right. \\
\left.\left.D u+(p-1)\left(\frac{u}{v}\right)^{p} D v\right)\right] d x= \\
=\int_{B}\left(v^{p}-u^{p}\right)\left[b+\frac{f}{v^{p-1}}\right] d x .
\end{gathered}
$$

It is trivial to see that the sum of the second members of (12) and (13) is less than or equal to zero, so, adding term by term equalities (12) and (13) we obtain:

$$
\begin{aligned}
\int_{B} u^{p}\left(|D(\log u)|^{p}-p|D(\log v)|^{p-2} D(\log v)\right. & D(\log u)+ \\
& \left.+(p-1)|D(\log v)|^{p}\right) d x+ \\
+\int_{B} v^{p}\left(|D(\log v)|^{p}-p|D(\log u)|^{p-2} D(\log u)\right. & D(\log v)+ \\
& \left.+(p-1)|D(\log u)|^{p}\right) d x \leq 0 .
\end{aligned}
$$


From (14), according to Lemma 2.3, if $p \geq 2$, it follows that:

$$
\int_{B}\left(u^{p}+v^{p}\right) \frac{|D(\log u)-D(\log v)|^{p}}{\left(2^{p-1}-1\right)} d x \leq 0,
$$

and, if $1<p<2$, there exists a positive constant $h(p)$ such that:

$$
\int_{B}\left(u^{p}+v^{p}\right) h(p) \frac{|D(\log u)-D(\log v)|^{2}}{(|D(\log u)|+|D(\log v)|)^{2-p}} d x \leq 0 .
$$

Consequently, from (15) and (16) it follows, for $p>1$, that $|D(\log u)-D(\log v)|=0$ a. e. in $B$. Then, there exists a constant $a>0$ such that $u=a v$ a. e. in $B$. Thus, being $u$ and $v$ solutions of $(9)$ and $|\{x \in B: f(x) \neq 0\}|>0$, it follows that $u=v$ a. e. in $B$. The uniqueness is thus proved.

An immediate consequence of uniqueness is that $v(x)=v(|x|)$. In order to prove completely (10), let us write $v(x)=\tilde{v}\left(\omega_{n}|x|^{n}\right)$ and set $s=\omega_{n}|x|^{n}$. Observing that $b(x)=\left(b^{+}\right)^{\#}-\left(b^{-}\right)_{\#}$, from (9) we obtain:

$$
-\left|\tilde{v}^{\prime}(s)\right|^{p-2} \tilde{v}^{\prime}(s)=\frac{s^{-(1-1 / n) p}}{\left(n \omega_{n}^{1 / n}\right)^{p}} \psi(s), \quad \text { in }(0,|B|),
$$

where $\psi(s)=\int_{0}^{s}\left[f^{*}(t)+\left(\left(b^{+}\right)^{*}(t)-\left(b^{-}\right)_{*}(t)\right)(\tilde{v}(t))^{p-1}\right] d t$.

To prove $(10)$ it is enough to show that in $(0,|B|)$ it results:

$$
\psi(s) \geq 0
$$

Set $s_{0}=\inf \left\{s \in[0,|B|]:\left(b^{-}\right)_{*}(s)>0\right\}\left(s_{0}=|B|\right.$ if $\left.b^{-} \equiv 0\right)$, we have:

$$
\psi(s)= \begin{cases}\int_{0}^{s}\left[f^{*}(t)+\left(b^{+}\right)^{*}(t)(\tilde{v}(t))^{p-1}\right] d t, & \forall s \in\left[0, s_{0}\right] \\ \psi\left(s_{0}\right)+\int_{s_{0}}^{s}\left[f^{*}(t)-\left(b^{-}\right)_{*}(t)(\tilde{v}(t))^{p-1}\right] d t, & \forall s \in\left[s_{0},|B|\right] .\end{cases}
$$

The condition (18) is obviously verified when $s \leq s_{0}$. So, let us suppose that there is an $s>s_{0}$ such that $\psi(s)<0$. Therefore, there exists $\left.\left.\bar{s} \in\right] s_{0},|B|\right]$ such that $\psi(\bar{s})=\min _{s \in\left[s_{0},|B|\right]} \psi(s)<0$. Clearly $\bar{s}<|B|$, otherwise from (17) it follows that $\tilde{v}^{\prime}(s)>0$ in some neighbourhood of $|B|$, in contrast with the fact that $\tilde{v}(s) \geq 0$ in $[0,|B|]$ and $\tilde{v}(|B|)=0$. Moreover, let us notice that there is an $s \in] \bar{s},|B|[$ such that $\psi(s)>0$. Indeed, if $\psi(s) \leq 0$ in $[\bar{s},|B|]$, from (17) it follows that $\tilde{v}(s)$ is increasing in $[\bar{s},|B|]$ from which, being $\tilde{v}(|B|)=0, \tilde{v}$ is equal to zero in $[\bar{s},|B|]$. So, $\psi(s)=0$ in $[\bar{s},|B|]$, but this is absurd.

Then, we can conclude that there exist $\left.s_{1}, s_{2} \in\right] s_{0},|B|\left[\right.$ such that $s_{1}<\bar{s}<s_{2}$, $\psi\left(s_{2}\right)=0, \psi(s)<0, \forall s \in\left[s_{1}, s_{2}[\right.$. In particular we have:

$$
\psi(\bar{s})=\min _{s \in\left[s_{1}, s_{2}\right]} \psi(s)
$$


As regards $\tilde{v}$ we can say that $\tilde{v}^{\prime}(s) \geq 0, \forall s \in\left[s_{1}, s_{2}\right]$, then the function $f^{*}(s)-\left(b^{-}\right)_{*}(s)(\tilde{v}(s))^{p-1}$ is decreasing in $\left[s_{1}, s_{2}\right]$, thus $\psi^{\prime}(s)$ is decreasing in $\left[s_{1}, s_{2}\right]$. So, $\psi(s)$ is concave in $\left[s_{1}, s_{2}\right]$, then $\psi(s)$ is constant in $\left[s_{1}, s_{2}\right]$, in contrast with the fact that $\psi(\bar{s})<0$ and $\psi\left(s_{2}\right)=0$.

The condition (10) is thus proved.

Remark 3.2. Let us consider $k \in] 0, \sup v\left[\right.$ and set $B_{k}=\{x \in B: v(x)>k\}$. If $v$ is a solution of (9) then $v$ is a solution of the following problem:

$$
\begin{cases}-\Delta_{p} v=b|v|^{p-2} v+f & \text { in } B_{k} \\ v=k & \text { on } \partial B_{k} .\end{cases}
$$

Moreover, $v$ satisfies the condition below:

$$
\begin{aligned}
& \frac{1}{p} \int_{B_{k}}|D v|^{p} d x-\frac{1}{p} \int_{B_{k}} b|v|^{p} d x-\int_{B_{k}} f v d x \leq \\
& \leq \frac{1}{p} \int_{B_{k}}|D w|^{p} d x-\frac{1}{p} \int_{B_{k}} b|w|^{p} d x-\int_{B_{k}} f w d x
\end{aligned}
$$

for all $w \in W^{1, p}\left(B_{k}\right)$ such that $w-k \in W_{0}^{1, p}\left(B_{k}\right)$. So, if $w=w^{\#}$ and $s=\omega_{n}|x|^{n}$, we have:

$$
\begin{aligned}
& \frac{\left(n \omega_{n}^{1 / n}\right)^{p}}{p} \int_{0}^{\left|B_{k}\right|} s^{(1-1 / n) p}\left|-\left(v^{*}\right)^{\prime}(s)\right|^{p} d s-\frac{1}{p} \int_{0}^{\left|B_{k}\right|} \tilde{b}(s)\left(v^{*}(s)\right)^{p} d s-\int_{0}^{\left|B_{k}\right|} f^{*}(s) v^{*}(s) d s \leq \\
& \leq \frac{\left(n \omega_{n}^{1 / n}\right)^{p}}{p} \int_{0}^{\left|B_{k}\right|} s^{(1-1 / n) p}\left|-\left(w^{*}\right)^{\prime}(s)\right|^{p} d s-\frac{1}{p} \int_{0}^{\left|B_{k}\right|} \tilde{b}(s)\left(w^{*}\right)^{p}(s) d s-\int_{0}^{\left|B_{k}\right|} f^{*}(s) w^{*}(s) d s
\end{aligned}
$$

where $b(x)=\tilde{b}\left(\omega_{n}|x|^{n}\right)$.

\section{Main result}

Let us consider the following Dirichlet problem:

$$
\begin{cases}-\operatorname{div}(a(x, u, D u))=g(x, u)+f & \text { in } \Omega \\ u=0 & \text { on } \partial \Omega .\end{cases}
$$


We assume that $a(x, s, \xi): \Omega \times \mathbf{R} \times \mathbf{R}^{n} \rightarrow \mathbf{R}^{n}$ and $g(x, s): \Omega \times \mathbf{R} \rightarrow \mathbf{R}$, are Carathéodory functions satisfying for some $p \in] 1,+\infty[$ the following conditions:

$$
\begin{aligned}
& |a(x, s, \xi)| \leq \alpha\left(|\xi|^{p-1}+|s|^{p-1}+k(x)\right), \text { a. e. } x \in \Omega, \forall(s, \xi) \in \mathbf{R} \times \mathbf{R}^{n}, \\
& a(x, s, \xi) \xi \geq|\xi|^{p}, \text { a. e. } x \in \Omega, \forall(s, \xi) \in \mathbf{R} \times \mathbf{R}^{n}, \\
& g(x, s) s \leq c(x)|s|^{p}, \text { a. e. } x \in \Omega, \forall s \in \mathbf{R}, \\
& |g(x, s)| \leq \beta|s|^{p-1}, \text { a. e. } x \in \Omega, \forall s \in \mathbf{R},
\end{aligned}
$$

where $c \in L^{\infty}(\Omega), \alpha>0, \beta>0$ and $k \geq 0$ is such that $k \in L^{p^{\prime}}(\Omega)$.

The present section is devoted to the comparison result between the decreasing rearrangement $u^{*}$ of a solution $u \in W_{0}^{1, p}(\Omega)$ of the problem (19) and the decreasing rearrangement $v^{*}$ of the solution $v \in W_{0}^{1, p}\left(\Omega^{\#}\right)$ of the problem:

$$
\begin{cases}-\Delta_{p} v=\hat{c}|v|^{p-2} v+f^{\#} & \text { in } \Omega^{\#} \\ v=0 & \text { on } \partial \Omega^{\#}\end{cases}
$$

where

$$
\hat{c}(x)=\left(c^{+}\right)^{\#}(x)-\left(c^{-}\right) \#(x), \quad \forall x \in \Omega^{\#} .
$$

Theorem 4.1. Let $u \in W_{0}^{1, p}(\Omega)$ be a solution of problem (19), under the assumptions $(20) \div(23)$ and $f \in L^{\infty}(\Omega)$. If c satisfies (7) and $v \in W_{0}^{1, p}\left(\Omega^{\#}\right)$ is the solution of problem (24), we have:

$$
u^{*}(s) \leq v^{*}(s), \quad \forall s \in\left[0, s_{0}\right]
$$

and

$$
\int_{0}^{s}\left(u^{*}(t)\right)^{p-1} d t \leq \int_{0}^{s}\left(v^{*}(t)\right)^{p-1} d t, \quad \forall s \in\left[s_{0},|\Omega|\right],
$$

where $s_{0}=\inf \left\{s \in[0,|\Omega|]:\left(c^{-}\right)_{*}(s)>0\right\} \quad\left(s_{0}=|\Omega|\right.$ if $\left.c^{-} \equiv 0\right)$.

To prove Theorem 4.1 we need three lemmas. Before stating them we introduce some notations.

Let $\tilde{c}(s)=\left(c^{+}\right)^{*}(s)-\left(c^{-}\right)_{*}(s), s \in[0,|\Omega|]$, and:

$$
\begin{array}{ll}
U(s)=\int_{0}^{s} \tilde{c}(t)\left(u^{*}(t)\right)^{p-1} d t, \quad \forall s \in[0,|\Omega|], \\
U_{+}(s)=\int_{0}^{s}\left(c^{+}\right)^{*}(t)\left(u^{*}(t)\right)^{p-1} d t, \quad \forall s \in\left[0, s_{0}\right], \\
U_{-}(s)=\int_{0}^{s}\left(c^{-}\right)_{*}(t)\left(u^{*}(t)\right)^{p-1} d t, \quad \forall s \in\left[s_{0},|\Omega|\right],
\end{array}
$$

where $s_{0}$ is defined in Theorem 4.1 . 
Analogously, we define $V, V_{+}, V_{-}$, related to $v^{*}$. Moreover, let us set:

$$
F(s)=\int_{0}^{s} f^{*}(t) d t, \quad \forall s \in[0,|\Omega|],
$$

and $\gamma(s)=\frac{s^{-(1-1 / n) p^{\prime}}}{\left(n \omega_{n}^{1 / n}\right) p^{\prime}}$, where $p^{\prime}=\frac{p}{p-1}$.

Lemma 4.2. Under the hypotheses of Theorem 4.1, we have, a. e. in $(0,|\Omega|)$, that:

$$
\begin{aligned}
& \left(-u^{*}(s)\right)^{\prime} \leq \gamma(s)[F(s)+U(s)]^{1 /(p-1)}, \\
& \left(-v^{*}(s)\right)^{\prime}=\gamma(s)[F(s)+V(s)]^{1 /(p-1)} .
\end{aligned}
$$

Proof. The demonstration of this lemma is similar to the one of the Lemma 3.2 of [11]. In this case, being $c(x)=\left(c^{+}\right)(x)-\left(c^{-}\right)(x)$, we just have to observe that:

$$
\int_{|u|>t} c(x)|u(x)|^{p-1} d x \leq \int_{0}^{\mu_{u}(t)}\left[\left(c^{+}\right)^{*}(s)-\left(c^{-}\right)_{*}(s)\right]\left(u^{*}(s)\right)^{p-1} d s .
$$

Lemma 4.3. Under the hypotheses of Theorem 4.1, with $f \not \equiv 0$, the following inequality holds:

$$
U_{+}(s) \leq V_{+}(s), \quad \forall s \in\left[0, s_{0}\right]
$$

Proof. Inequality (29) is trivial when $c^{+} \equiv 0$, so, from now on, we suppose $c^{+} \not \equiv 0$.

Let us distinguish two different cases:

1) $U_{+}\left(s_{0}\right) \leq V_{+}\left(s_{0}\right)$,

2) $U_{+}\left(s_{0}\right)>V_{+}\left(s_{0}\right)$.

Let us consider the case 1$)$. If (29) is not satisfied, then there exists $\bar{s} \in] 0, s_{0}[$ such that:

$$
U_{+}(\bar{s})-V_{+}(\bar{s})=\max _{s \in\left[0, s_{0}\right]}\left(U_{+}(s)-V_{+}(s)\right)>0 .
$$

Let us set:

$$
\begin{aligned}
& s_{1}=\inf \left\{s \in[0, \bar{s}]: U_{+}(t)>V_{+}(t), \forall t \in[s, \bar{s}]\right\}, \\
& s_{2}=\sup \left\{s \in\left[\bar{s}, s_{0}\right]: U_{+}(t)>V_{+}(t), \forall t \in[\bar{s}, s]\right\} .
\end{aligned}
$$

Observing that $U^{+} / V^{+}$and $V^{+} / U^{+}$are bounded in $\left[s_{1}, s_{2}\right]$, we can use the following test functions:

$$
\varphi_{1}(s)=\frac{\left(U_{+}(s)\right)^{p^{\prime}}-\left(V_{+}(s)\right)^{p^{\prime}}}{\left(U_{+}(s)\right)^{p^{\prime}-1}},
$$




$$
\varphi_{2}(s)=\frac{\left(U_{+}(s)\right)^{p^{\prime}}-\left(V_{+}(s)\right)^{p^{\prime}}}{\left(V_{+}(s)\right)^{p^{\prime}-1}} .
$$

Inserting $\varphi_{1}$ and $\varphi_{2}$ in the relations (27) and (28), respectively, and integrating between $s_{1}$ and $s_{2}$, we have:

$$
\int_{s_{1}}^{s_{2}}\left(-u^{*}(s)\right)^{\prime} \varphi_{1}(s) d s \leq \int_{s_{1}}^{s_{2}} \gamma(s)\left(\left(U_{+}(s)\right)^{p^{\prime}}-\left(V_{+}(s)\right)^{p^{\prime}}\right)\left(\frac{F(s)}{U_{+}(s)}+1\right)^{p^{\prime}-1} d s
$$

and:

$$
\int_{s_{1}}^{s_{2}}\left(-v^{*}(s)\right)^{\prime} \varphi_{2}(s) d s=\int_{s_{1}}^{s_{2}} \gamma(s)\left(\left(U_{+}(s)\right)^{p^{\prime}}-\left(V_{+}(s)\right)^{p^{\prime}}\right)\left(\frac{F(s)}{V_{+}(s)}+1\right)^{p^{\prime}-1} d s .
$$

Being $U_{+}(s)>V_{+}(s)$ in $] s_{1}, s_{2}$ [, we have that the difference of the second members of (30) and (31) is less than zero, so:

$$
\int_{s_{1}}^{s_{2}}\left(-u^{*}(s)\right)^{\prime} \varphi_{1}(s) d s+\int_{s_{1}}^{s_{2}}\left(v^{*}(s)\right)^{\prime} \varphi_{2}(s) d s<0 .
$$

Integrating by parts the first member of (32) and bearing in mind that $U_{+}\left(s_{1}\right)-$ $V_{+}\left(s_{1}\right)=U_{+}\left(s_{2}\right)-V_{+}\left(s_{2}\right)=0$, we have:

$$
\int_{s_{1}}^{s_{2}}\left[u^{*}(s)\left(\varphi_{1}(s)\right)^{\prime}-v^{*}(s)\left(\varphi_{2}(s)\right)^{\prime}\right] d s<0 .
$$

Being:

$$
\left(\varphi_{1}\right)^{\prime}=\left(c^{+}\right)^{*}\left(u^{*}\right)^{p-1}+\left(p^{\prime}-1\right)\left(c^{+}\right)^{*}\left(u^{*}\right)^{p-1}\left(\frac{V_{+}}{U_{+}}\right)^{p^{\prime}}-p^{\prime}\left(c^{+}\right)^{*}\left(v^{*}\right)^{p-1}\left(\frac{V_{+}}{U_{+}}\right)^{p^{\prime}-1},
$$

and:

$\left(\varphi_{2}\right)^{\prime}=-\left(c^{+}\right)^{*}\left(v^{*}\right)^{p-1}-\left(p^{\prime}-1\right)\left(c^{+}\right)^{*}\left(v^{*}\right)^{p-1}\left(\frac{U_{+}}{V_{+}}\right)^{p^{\prime}}+p^{\prime}\left(c^{+}\right)^{*}\left(u^{*}\right)^{p-1}\left(\frac{U_{+}}{V_{+}}\right)^{p^{\prime}-1}$, then, the first member of (33) is equal to:

$$
\begin{aligned}
\int_{s_{1}}^{s_{2}}\left(c^{+}\right)^{*}\left[\left(U_{+}\right)^{p^{\prime}}\left(\frac{\left(u^{*}\right)^{p}}{\left(U_{+}\right)^{p^{\prime}}}-p^{\prime} \frac{\left(u^{*}\right)^{p-1}}{U_{+}} \frac{v^{*}}{\left(V_{+}\right)^{p^{\prime}-1}}+\left(p^{\prime}-1\right) \frac{\left(v^{*}\right)^{p}}{\left(V_{+}\right)^{p^{\prime}}}\right)+\right. \\
\left.+\left(V_{+}\right)^{p^{\prime}}\left(\frac{\left(v^{*}\right)^{p}}{\left(V_{+}\right)^{p^{\prime}}}-p^{\prime} \frac{\left(v^{*}\right)^{p-1}}{V_{+}} \frac{u^{*}}{\left(U_{+}\right)^{p^{\prime}-1}}+\left(p^{\prime}-1\right) \frac{\left(u^{*}\right)^{p}}{\left(U_{+}\right)^{p^{\prime}}}\right)\right] d s .
\end{aligned}
$$

Then, set $x=\frac{\left(u^{*}\right)^{\frac{p}{p^{\prime}}}}{U_{+}}$and $y=\frac{\left(v^{*}\right)^{\frac{p}{p^{\prime}}}}{V_{+}}$, according to Lemma 2.3 , if $p^{\prime} \geq 2$ it follows that the left hand side of (33) is greater than or equal to:

$$
\int_{s_{1}}^{s_{2}}\left(c^{+}\right)^{*}\left(\left(U_{+}\right)^{p^{\prime}}+\left(V_{+}\right)^{p^{\prime}}\right) \frac{|y-x|^{p^{\prime}}}{2^{p^{\prime}-1}-1} d s
$$


and, if $1<p^{\prime}<2$, there exists a positive constant $h\left(p^{\prime}\right)$ such that the left hand side of (33) is greater than or equal to:

$$
h\left(p^{\prime}\right) \int_{s_{1}}^{s_{2}}\left(c^{+}\right)^{*}\left(\left(U_{+}\right)^{p^{\prime}}+\left(V_{+}\right)^{p^{\prime}}\right) \frac{|y-x|^{2}}{(|x|+|y|)^{2-p^{\prime}}} d s .
$$

Consequently, being (34) and (35) greater than or equal to zero, from (33) we have an absurd. So, condition $(29)$ is verified if $U_{+}\left(s_{0}\right) \leq V_{+}\left(s_{0}\right)$.

Now, let us consider the case 2$)$.

If $u^{*}\left(s_{0}\right) \leq v^{*}\left(s_{0}\right)$, set:

$$
s_{1}=\inf \left\{s \in\left[0, s_{0}\right]: U_{+}(t)>V_{+}(t), \forall t \in\left[s, s_{0}\right]\right\},
$$

let us observe that $U_{+}\left(s_{1}\right)=V_{+}\left(s_{1}\right)$; moreover, being $u^{*}\left(s_{0}\right) \leq v^{*}\left(s_{0}\right)$ and $U_{+}\left(s_{0}\right)>$ $V_{+}\left(s_{0}\right)$, we have:

$$
\left(-\frac{u^{*}\left(s_{0}\right)}{\left(U_{+}\left(s_{0}\right)\right)^{p^{\prime}-1}}+\frac{v^{*}\left(s_{0}\right)}{\left(V_{+}\left(s_{0}\right)\right)^{p^{\prime}-1}}\right)\left(\left(U_{+}\left(s_{0}\right)\right)^{p^{\prime}}-\left(V_{+}\left(s_{0}\right)\right)^{p^{\prime}}\right) \geq 0 .
$$

Then, we can proceed as in case 1). Inserting $\varphi_{1}$ and $\varphi_{2}$ in the relations (27) and (28), respectively, and integrating between $s_{1}$ and $s_{0}$, we obtain an absurd.

If $u^{*}\left(s_{0}\right)>v^{*}\left(s_{0}\right)$, set:

$$
\overline{\bar{s}}=\sup \left\{s \in\left[s_{0},|\Omega|\right]: u^{*}(t)>v^{*}(t), \forall t \in\left[s_{0}, s\right]\right\},
$$

let us distinguish the following two cases:

2a) $U(\overline{\bar{s}}) \geq V(\overline{\bar{s}})$.

2b) $U(\overline{\bar{s}})<V(\overline{\bar{s}})$.

In the case $2 \mathrm{a}$ ), being:

$$
\frac{d}{d s}(U(s)-V(s))=\left(c^{-}\right)_{*}(s)\left(-\left(u^{*}\right)^{p-1}(s)+\left(v^{*}\right)^{p-1}(s)\right)<0, \quad \text { a. e. in }\left[s_{0}, \overline{\bar{s}}\right],
$$

we have that $U(s)-V(s)$ is decreasing in $\left[s_{0}, \overline{\bar{s}}\right]$. Thus, being $U(\overline{\bar{s}})-V(\overline{\bar{s}}) \geq 0$, it follows:

$$
U(s) \geq V(s), \quad \forall s \in\left[s_{0}, \overline{\bar{s}}\right] .
$$

Now, let us consider the following function:

$$
w(s)=\max \left\{u^{*}(s), v^{*}(s)\right\}, \quad \forall s \in[0, \overline{\bar{s}}] .
$$

Set $W(s)=\int_{0}^{s} \tilde{c}(t)(w(t))^{p-1} d t, \forall s \in[0, \bar{s}]$, it is easy to show that $w(s)$ satisfies the following relation:

$$
-w^{\prime}(s) \leq \gamma(s)[F(s)+W(s)]^{\frac{1}{p-1}}, \quad \forall s \in[0, \overline{\bar{s}}] .
$$


We also observe that a simple calculation gives:

$$
W(s) \geq U(s), \quad \forall s \in\left[s_{0}, \overline{\bar{s}}\right] .
$$

Moreover, because $u^{*}\left(s_{0}\right)>v^{*}\left(s_{0}\right)$, we have $v^{*} \not \equiv w$. Then, bearing in mind Remark 3.2 , as $v$ is the unique solution of the radial problem (24), we have:

$$
\begin{aligned}
& \frac{1}{p} \int_{0}^{\overline{\bar{s}}}\left(n \omega_{n}^{1 / n}\right)^{p} s^{(1-1 / n) p}\left|-\left(v^{*}(s)\right)^{\prime}\right|^{p} d s-\frac{1}{p} \int_{0}^{\overline{\bar{s}}} \tilde{c}(s)\left(v^{*}(s)\right)^{p} d s-\int_{0}^{\overline{\bar{s}}} f^{*}(s) v^{*}(s) d s< \\
& <\frac{1}{p} \int_{0}^{\overline{\bar{s}}}\left(n \omega_{n}^{1 / n}\right)^{p} s^{(1-1 / n) p}\left|-w^{\prime}(s)\right|^{p} d s-\frac{1}{p} \int_{0}^{\overline{\bar{s}}} \tilde{c}(s) w^{p}(s) d s-\int_{0}^{\bar{s}} f^{*}(s) w(s) d s .
\end{aligned}
$$

On the other hand, from (28) and (37), respectively, it follows:

$$
\begin{aligned}
& \left|-\left(v^{*}(s)\right)^{\prime}\right|^{p}=-\left(v^{*}(s)\right)^{\prime}\left(n \omega_{n}^{1 / n}\right)^{-p} s^{(1-1 / n)(-p)}[F(s)+V(s)], \\
& \left|-(w(s))^{\prime}\right|^{p} \leq-(w(s))^{\prime}\left(n \omega_{n}^{1 / n}\right)^{-p} s^{(1-1 / n)(-p)}[F(s)+W(s)] .
\end{aligned}
$$

Substituting (40) and (41) in (39) and integrating by parts between 0 and $\overline{\bar{s}}$, from (36) and (38) we obtain:

$$
\int_{0}^{\overline{\bar{s}}} v^{*}(s) f^{*}(s) d s>\int_{0}^{\overline{\bar{s}}} w(s) f^{*}(s) d s,
$$

but this is absurd because $v^{*}(s) \leq w(s)$ in $[0, \overline{\bar{s}}]$.

Finally, let us examine the case 2b). Being $u^{*}(s)>v^{*}(s)$ in $\left[s_{0}, \overline{\bar{s}}[\right.$, we have:

$$
\frac{d}{d s}(U(s)-V(s))<0, \quad \text { a. e. in }\left[s_{0}, \overline{\bar{s}}[,\right.
$$

then, $U(s)-V(s)$ is decreasing in $\left[s_{0}, \overline{\bar{s}}\right]$. So, there is $\left.\tilde{s} \in\right] s_{0}, \overline{\bar{s}}[$ such that:

$$
U(\tilde{s})=V(\tilde{s}) \quad \text { and } \quad U(s)<V(s), \quad \forall s \in] \tilde{s}, \overline{\bar{s}}] .
$$

As $\tilde{s} \in] s_{0}, \overline{\bar{s}}[$ thence:

$$
u^{*}(\tilde{s})>v^{*}(\tilde{s}) .
$$

On the other hand, integrating (27) and (28) between $\tilde{s}$ and $\overline{\bar{s}}$ and using (42) we have:

$$
u^{*}(\tilde{s})-u^{*}(\overline{\bar{s}})<v^{*}(\tilde{s})-v^{*}(\overline{\bar{s}}),
$$

in contrast with (43) because $u^{*}(\overline{\bar{s}})=v^{*}(\overline{\bar{s}})$. The case 2b) is absurd, too.

Lemma 4.4. Under the hypotheses of Theorem 4.1, with $f \not \equiv 0$, the following inequality holds:

$$
U_{-}(s) \leq V_{-}(s), \quad \forall s \in\left[s_{0},|\Omega|\right]
$$


Proof. Let us suppose that (44) is not satisfied. Let us set $Z(s)=U_{-}(s)-V_{-}(s)$, $\forall s \in\left[s_{0},|\Omega|\right]$. Let us observe that $Z\left(s_{0}\right)=0, Z^{\prime}(|\Omega|)=0$ and, moreover, there exists an $\left.\bar{s} \in] s_{0},|\Omega|\right]$ such that $Z(\bar{s})=\max _{s \in\left[s_{0},|\Omega|\right]} Z(s)>0$.

If $\bar{s}=|\Omega|$, let us consider:

$$
s_{1}=\inf \left\{s \in\left[s_{0},|\Omega|\right]: Z(t)>0, \forall t \in[s,|\Omega|]\right\} .
$$

Obviously, we have $Z\left(s_{1}\right)=0$ and $\left.\left.Z(s)>0, \forall s \in\right] s_{1},|\Omega|\right]$. Then, considering $\left.s \in] s_{1},|\Omega|\right]$ and integrating the relations (27) and (28) between $s$ and $|\Omega|$, by Lemma 4.3 we obtain:

$$
\begin{aligned}
u^{*}(s) & \leq \int_{s}^{|\Omega|}\left[\gamma(t)(F(t)+U(t))^{\frac{1}{p-1}}\right] d t=\int_{s}^{|\Omega|}\left[\gamma(t)\left(F(t)+U_{+}\left(s_{0}\right)-U_{-}(t)\right)^{\frac{1}{p-1}}\right] d t \leq \\
& \leq \int_{s}^{|\Omega|}\left[\gamma(t)\left(F(t)+V_{+}\left(s_{0}\right)-V_{-}(t)\right)^{\frac{1}{p-1}}\right] d t=\int_{s}^{|\Omega|}\left(-v^{*}(t)\right)^{\prime} d t=v^{*}(s) .
\end{aligned}
$$

So, because $s$ is a generic element of $\left.] s_{1},|\Omega|\right]$, we have:

$$
\left.\left.U_{-}(s) \leq V_{-}(s), \quad \forall s \in\right] s_{1},|\Omega|\right],
$$

but this is absurd because $\left.Z(s)>0, \forall s \in] s_{1},|\Omega|\right]$.

Now, let us consider the case in which $\bar{s} \in] s_{0},|\Omega|[$. This time we can consider $s_{1}, s_{2} \in\left[s_{0},|\Omega|\right]$ such that $s_{1}<\bar{s} \leq s_{2}$ and $Z\left(s_{1}\right)=0, Z^{\prime}\left(s_{2}\right) \leq 0, Z(s)>0$, $\left.\forall s \in] s_{1}, s_{2}\right]$. So, $U_{-}\left(s_{1}\right)=V_{-}\left(s_{1}\right)$ and, moreover:

$$
\left.\left.U_{-}(s)>V_{-}(s), \quad \forall s \in\right] s_{1}, s_{2}\right]
$$

Then, integrating (27) and (28) between $s$ and $s_{2}$ and, arguing as above, we have:

$$
\left.\left.u^{*}(s)-u^{*}\left(s_{2}\right)<v^{*}(s)-v^{*}\left(s_{2}\right), \quad \forall s \in\right] s_{1}, s_{2}\right] .
$$

Thus, being $Z^{\prime}\left(s_{2}\right) \leq 0$, it follows $\left.\left.u^{*}(s) \leq v^{*}(s), \forall s \in\right] s_{1}, s_{2}\right]$, and this implies:

$$
\left.\left.U_{-}(s) \leq V_{-}(s), \quad \forall s \in\right] s_{1}, s_{2}\right]
$$

in contrast with (46).

Proof of Theorem 4.1. If $f \equiv 0$ we are in a trivial case. Indeed, it is easy to prove that:

$$
u^{*}(s)=v^{*}(s)=0, \quad s \in[0,|\Omega|] .
$$

As regards $v$, we can use $v^{+}$and $v^{-}$in (24) and argue as in Theorem 3.1. As regards $u$, we can similarly use $u^{+}$in (19), obtaining:

$$
\int_{\Omega} a(x, u, D u) D u^{+} d x=\int_{\Omega} g(x, u) u^{+} d x .
$$


Assumptions (21), (22), (7) give us:

$$
\int_{\Omega}\left|D u^{+}\right|^{p} d x<\lambda\left(\Omega^{\#}\right) \int_{\Omega}\left|u^{+}\right|^{p} d x .
$$

On the other hand, it is well known that Faber-Krahn inequality for the first eigenvalue states (see [1]) that $\lambda\left(\Omega^{\#}\right) \leq \lambda(\Omega)$. Thus (47) implies $u^{+}=0$. A similar argument gives $u^{-}=0$.

From now on we suppose $f \not \equiv 0$ and use lemmas $4.2,4.3,4.4$. Let us observe that, from (44) and Lemma 2.1 we have:

$$
\int_{s_{0}}^{s}\left(u^{*}(t)\right)^{p-1} d t \leq \int_{s_{0}}^{s}\left(v^{*}(t)\right)^{p-1} d t, \quad \forall s \in\left[s_{0},|\Omega|\right] .
$$

So, from Lemma 2.2 we obtain:

$$
u^{*}\left(s_{0}\right) \leq v^{*}\left(s_{0}\right) .
$$

On the other hand, from (27), (28) and (29), we have:

$$
u^{*}(s)-u^{*}\left(s_{0}\right) \leq v^{*}(s)-v^{*}\left(s_{0}\right), \quad \forall s \in\left[0, s_{0}\right] .
$$

Then, from (49) we obtain (25).

Finally, the condition (26) easily follows from (25) and (48).

Acknowledgements. The support of GNAMPA-INdAM Progetto "Proprietà analiticogeometriche di soluzioni di equazioni ellittiche e paraboliche" (2002/2003) is acknowledged by the authors.

\section{References}

[1] A. Alvino, V. Ferone, and G. Trombetti, On the properties of some nonlinear eigenvalues, SIAM J. Math. Anal. 29 (1998), 437-451 (electronic).

[2] A. Alvino, G. Trombetti, and P.-L. Lions, On optimization problems with prescribed rearrangements, Nonlinear Anal. 13 (1989), 185-220.

[3] _ Comparison results for elliptic and parabolic equations via Schwarz symmetrization, Ann. Inst. H. Poincaré Anal. Non Linéaire 7 (1990), 37-65.

[4] A. Anane, O. Chakrone, and J.-P. Gossez, Spectre d'ordre supérieur et problémes de nonrésonance, C. R. Acad. Sci. Paris Sér. I Math. 325 (1997), 33-36.

[5] C. Bandle, Isoperimetric inequalities and applications, Monographs and Studies in Mathematics, vol. 7, Pitman (Advanced Publishing Program), Boston, Mass., 1980, ISBN 0-273-08423-2.

[6] M. Belloni and B. Kawohl, A direct uniqueness proof for equations involving the p-Laplace operator, Manuscripta Math. 109 (2002), 229-231. 
[7] M. F. Betta, V. Ferone, and A. Mercaldo, Regularity for solutions of nonlinear elliptic equations, Bull. Sci. Math. 118 (1994), 539-567.

[8] G. Chiti, Orlicz norms of the solutions of a class of elliptic equations, Boll. Un. Mat. Ital. A (5) 16 (1979), 178-185. (Italian, with English summary)

[9] J. I. Diaz, Symmetrization of nonlinear elliptic and parabolic problems and applications: a particular overview, Progress in Partial Differential Equations: Elliptic and Parabolic Problems (Pont-à-Mousson, 1991), Pitman Res. Notes Math. Ser., vol. 266, Longman Sci. Tech., Harlow, 1992, pp. 1-16.

[10] J. I. Díaz and J. E. Saá, Existence et unicité de solutions positives pour certaines équations elliptiques quasilinéaires, C. R. Acad. Sci. Paris Sér. I Math. 305 (1987), 521-524. (French, with English summary)

[11] V. Ferone and B. Messano, Comparison results for nonlinear elliptic equations with lower-order terms, Math. Nachr. 252 (2003), 43-50.

[12] W. H. Fleming and R. Rishel, An integral formula for total gradient variation, Arch. Math. 11 (1960), 218-222

[13] G. H. Hardy, J. L. Littlewood, and G. Pólya, Inequalities, Cambridge Univ. Press, 1964.

[14] B. Kawohl, Rearrangements and convexity of level sets in PDE, Lecture Notes in Mathematics, vol. 1150, Springer-Verlag, Berlin, 1985, ISBN 3-540-15693-3.

[15] P. Lindqvist, On the equation $\operatorname{div}\left(|\nabla u|^{p-2} \nabla u\right)+\lambda|u|^{p-2} u=0$, Proc. Amer. Math. Soc. 109 (1990), 157-164.

[16] V. G. Maz'ja, Weak solutions of the Dirichlet and Neumann problems, Trudy Moskov. Mat. Obšč. 20 (1969), 137-172. (Russian. English translation: Trans. Moscow Math. Soc.)

[17] J. Mossino, Inégalités isopérimétriques et applications en physique, Travaux en Cours. [Works in Progress], Hermann, Paris, 1984, ISBN 2-7056-5963-3.

[18] G. Talenti, Elliptic equations and rearrangements, Ann. Scuola Norm. Sup. Pisa Cl. Sci. (4) 3 (1976), 697-718

[19] Nonlinear elliptic equations, rearrangements of functions and Orlicz spaces, Ann. Mat. Pura Appl. (4) 120 (1979), 160-184.

[20] G. Trombetti and J. L. Vázquez, A symmetrization result for elliptic equations with lower-order terms, Ann. Fac. Sci. Toulouse Math. (5) 7 (1985), 137-150.

[21] J. L. Vázquez, A strong maximum principle for some quasilinear elliptic equations, Appl. Math. Optim. 12 (1984), 191-202.

[22] H. F. Weinberger, Symmetrization in uniformly elliptic problems, Studies in Mathematical Analysis and Related Topics, Stanford Univ. Press, Stanford, Calif., 1962, pp. 424-428. 